\title{
Editorial: Latest Developments in the Field of Magnesium Alloys and Their Applications
}

\author{
Hajo Dieringa ${ }^{1 *}$, David StJohn ${ }^{2}$, María Teresa Pérez Prado ${ }^{3}$ and Karl Ulrich Kainer ${ }^{4}$ \\ ${ }^{1}$ Helmholtz-Zentrum Hereon, Institute of Materials and Process Design, Department Hybrid Materials and Processes, \\ Geesthacht, Germany, ${ }^{2}$ School of Mechanical and Mining Engineering, The University of Queensland, Brisbane, QLD, Australia, \\ ${ }^{3}$ MDEA Materials Institute, Madrid, Spain, ${ }^{4}$ Wrocław University of Science and Technology, Wrocław, Poland
}

Keywords: lightweight, cast and wrought alloys, biodegradability, corrosion protection, creep resistance, hybrid materials, functionalization

Editorial on the Research Topic

Latest Developments in the Field of Magnesium Alloys and their Applications

Magnesium-based alloys and composites as structural materials as well as functionalized magnesium materials have been experiencing a steady increase in interest in the demand for new applications as well as in the scientific community for many years. To give an expression and visibility for this high demand, the authors initiated this Research Topic to provide interested stakeholders with an overview of current developments in research in this sector. A total of 16 articles were published by 56 authors from 29 institutional affiliations in nine countries. 12 of these articles are original research, and two of each are mini-reviews and reviews. Of the original research articles, eight address wrought alloys, two cast materials, and two fundamentals of metallurgical principles of magnesium alloys. This certainly meets the current need for optimization of wrought $\mathrm{Mg}$ materials for sheet applications or extruded profiles. In the list of applications targeted by the authors, it is noticeable that biodegradable magnesium materials are now a major focus. This is partly due to the fact that this research field experienced an acceleration in interest a few years ago and partly due to the massive interest of the medical industry in new, cost-effective solutions in implant materials to enable transfer to, for example, surgical procedures. The two mini-reviews deal firstly with the suitability assessment of magnesium materials for new applications in drone design and secondly with the deformation properties of friction-stir-welded magnesium alloys. The two reviews target fundamental issues of alloy development in magnesium alloys, as well as the suitability of magnesium materials as hydrogen storage materials.

In the first mini-review (Ren et al.), an overview of the microstructural characteristics and resulting texture development of magnesium alloys that were welded using the Friction-Stir process is presented. This process is a solid-phase joining process that does not suffer solidification problems as in other welding processes. EBSD data, among other data, are used for the analysis, and the localised plastic deformation mechanisms are described. In addition to these analyses, process modifications are also presented that serve to increase the strength of the welded zone.

The second mini-review addresses the suitability of magnesium-based materials as construction materials for drones (Höche et al.). The main drivers for this are low density and good castability, but also good damping properties. However, critical characteristics are also highlighted and ways to avoid them are presented. With the help of Ashby maps, which plot the Young's modulus or strength against the material's density, the fields of possible application are identified and some new monolithic but also hybrid magnesium materials are presented for further developments, which can be used in manned or unmanned aerial vehicles in the future. 
The first review (Rabkin et al.) deals with the hydrogen storage capability of extremely fine-grained magnesium alloys, one of the innovative topics in magnesium technology that fall under the category of functionalization. The fine grain size is achieved with the equal channel angular processing process (ECAP) and grain sizes below $1 \mu \mathrm{m}$ are achieved. Also, other severe plastic deformation processes (SPD) such as high pressure torsion (HPT) result in grain sizes that significantly improve the kinetics of hydrogen loading and deloading. The authors argue that the best way to optimize the thermodynamics of the $\mathrm{Mg}-\mathrm{H}$ system is to combine SPD with the selection of light alloying elements, which can be supported with computer-aided thermodynamic simulations (Campos et al.). Magnesium-based nanocomposites reinforced with carbon homologues, such as graphene or nanotubes, also show potential for suitability as hydrogen storage materials. More scientific studies are needed to better understand the detailed mechanisms of absorption and desorption and the influence of lattice defects.

The second review summarizes the basic principles of magnesium alloying (Kaya). In doing so, Miedema's model becomes the center of attention along with the assumptions according to Hume-Rothery. This Miedema assumption relates the electron density and the difference in electronegativity of two elements to the stacking fault energy generated thereby. In the simulation approaches, the short-range order (SRO) formation of atoms is considered and not only the uniform homogeneous distribution of atoms in solid solution. Furthermore, the author would like to see this Miedema approach extended to multicomponent systems. As examples, oxygen and $\mathrm{Ca}$ or $\mathrm{CaO}$ are presented here as alloying additions. This review article describes in detail the possibilities for modelling magnesium alloy systems and attempts to derive property predictions from the results. It will be a good basis for future alloy developments and alloy innovations.

In an original research article, Tun et al. developed and tested a high-entropy alloy (HEA) with composition $\mathrm{Mg}$-xAl-xCu-xMnxZn with $x=5$ at\% (Tun et al.). This material was extruded and $\mathrm{Al6Mn}$ and $\mathrm{Al} 2 \mathrm{CuMg}$ phases appear in the microstructure, resulting in the high hardness combined with high compressive strength of $616 \mathrm{MPa}$ also achieving good ductility of $16.9 \%$. The tensile strength and ductility in the tensile test are found to be good at $318 \mathrm{MPa}$ and $8.2 \%$, respectively. Regarding the yield strength, the ratio between compression and tensile tests is 0.9 , which is almost symmetrical and thus quite untypical for magnesium alloys.

In an original research article, the microstructure, texture, as well as the forming behaviour and mechanical characteristics of two magnesium alloys (Mg-Zn- $\mathrm{RE}$ and $\mathrm{Mg}-\mathrm{Zn}-\mathrm{Ca})$ produced by twin roll casting process were investigated (VictoriaHernández et al.). Both alloys were hot rolled and annealed. They both show a fine-grained microstructure, a good ductility higher than $30 \%$, and a similar texture with a split in the transverse direction. However, the difference in yield strength in and perpendicular to the rolling direction is relatively large. The Erichsen Index (IE) of the Mg-Zn-RE is 8.4 after cold rolling, while the $\mathrm{Mg}-\mathrm{Zn}-\mathrm{Ca}$ alloy shows good stretch formability while having a lower Erichsen Index. The alloy containing calcium does not show exceptional hot formability.
The crack-inducing effect of the $\mathrm{Mg}_{2} \mathrm{Ca}$ phases is probably responsible for this. In conclusion, the suppression of the split texture is an effective way to suppress the anisotropy of the mechanical properties.

The effect of the addition of an Al-Be master alloy on the grain refining of a $\mathrm{Mg}$-Zr alloy is studied in an original research article (Balasubramani et al.). It was originally assumed that the grain coarsening effect of this addition was due to a reaction of Be with $\mathrm{Zr}$, thereby reducing the grain refining potential of the zirconium. In this work, however, it was shown that a reaction of the $\mathrm{Al}$ with $\mathrm{Zr}$ leads to intermetallic phases and thus reduces the absolute concentration of dissolved Zr. Consequently, this leads to a reduction of the grain refining effect by Zr. Furthermore, the grain refining effect of ultrasonic treatment of the melt was demonstrated and it was shown that beryllium is linked to oxides formed at the surface. The results of this study show that $\mathrm{Be}$ additions can lead to a reduction of ignitibility in grain refined cast alloys.

Creep resistance under compressive stress is investigated in an original research article on seven commercial and newly developed creep-resistant magnesium alloys processed in highpressure die casting (Gavras et al.). These are the commercial AE42 and AE44, as well as a modified AE44, a commercial MRI230D, and three newly developed DieMag alloys that contain aluminium as well as barium and calcium in a 2:1:1 ratio in varying concentrations. In tensile tests at room temperature (RT), DieMag633 showed the highest yield strength of more than $200 \mathrm{MPa}$, but at the expense of ductility. MRI230D exhibited the second highest yield strength at RT with $180 \mathrm{MPa}$, followed by DieMag422 with $173 \mathrm{MPa}$. In tensile tests at $150^{\circ} \mathrm{C}$, DieMag633 was also convincing with $160 \mathrm{MPa}$ followed by MRI230D and DieMag422 with around $140 \mathrm{MPa}$. Thus, the yield strength of this most creep-resistant magnesium alloy at RT and at $150^{\circ} \mathrm{C}$ is higher than those of the aluminium alloy $\mathrm{A} 380$. The creep resistance at $200^{\circ} \mathrm{C}$ and different stresses between 60 and $100 \mathrm{MPa}$ was best in the lower stress range for AE44 and at higher stresses for DieMag633.

The corrosion rate and mechanical properties of $\mathrm{Mg}$-xAg alloys with $\mathrm{x}$ between 2 and $10 \mathrm{wt} \%$, which may be suitable as biodegradable materials, are investigated in an original research article (Jessen et al.). These are thin films prepared by the magnetron sputtering technique and are as thin as $20 \mu \mathrm{m}$. They are compared with samples of pure magnesium produced in an identical manner. It was found that a $\mathrm{Mg}$ $10 \mathrm{Ag}$ alloy exhibits a tensile strength of $371 \mathrm{MPa}$ with, however, moderate ductility. At a content of $6 \mathrm{wt} \%$ silver, the material shows a yield strength of $310 \mathrm{MPa}$ with an elongation of $6 \%$. The simultaneous investigation of the corrosion rates in combination with the mechanical properties suggest that the Mg-6Ag material is a promising material for degradable implants because the silver can also exhibit therapeutic effects.

Another original research paper addresses the biodegradable magnesium materials $\mathrm{Mg}$-xGd with $X=2,5$ and 10 , which can be used as implant materials (Harmuth et al.). The alloys were cast, solution treated and then extruded at different temperatures and speeds and the obtained profiles were investigated for their properties. It is shown that the mechanical properties can be 
varied over a wide range by the appropriate choice of parameters. Thus, the yield strengths in the tensile test range from 90 to $200 \mathrm{MPa}$ and the ultimate tensile strengths from 180 to $280 \mathrm{MPa}$. In compression, yield strengths are between 80 and $220 \mathrm{MPa}$ and ultimate compressive strengths between 300 and $450 \mathrm{MPa}$. Degradation rates are low at about $0.2 \mathrm{~mm} /$ year for all alloys and are not affected by process parameters. Mg-Gd alloys are therefore suitable for use as degradable implant materials.

Compression tests on a rolled and annealed Mg-1Zn-1Gd$0.6 \mathrm{Zr}$ alloy (ZEK110), which were performed in the sheet plane in the rolling direction, showed the following characteristics after analysis of the microstructure and texture of the deformed specimens (Basu and Al-Samman). Microstructure after annealing showed a large number of compression twins but also tension twins, which furthermore included second and third order twins. However, the growth of the twins is strongly hindered by $\mathrm{Zr}$ rich precipitates and stress fields around other particles. $<\mathrm{c}+\mathrm{a}>$ slip incorporates local plastic shear across the twin boundaries, while basal slip accumulates at interfaces in local dislocation pileup, contributing to grain boundary strengthening. Recrystallization and grain growth takes place. The importance of understanding twin deformation for the overall deformation process in magnesium materials has been demonstrated.

Another original research paper, which deals with the property optimisation of a $\mathrm{Mg}-3 \mathrm{Zn}-0.2 \mathrm{Ca}$ alloy by adding manganese (between 0.3 and $0.9 \mathrm{wt} \%$ ), sees biomedicine as an application area for these alloys and, in particular, their use as degradable implant materials (Han et al.). With the increase of manganese, the grain size of the cast material first decreases and then increases again. This effect is attributed to the influence of manganese on supercooling during the solidification process. After extrusion of the materials, it is shown that with a content of $0.5 \mathrm{wt} \%$ manganese, a maximum yield strength of $302 \mathrm{MPa}$ and a ultimate tensile strength of $327 \mathrm{MPa}$ can be achieved. This is attributed to the influence of manganese on the recrystallization behaviour, which in turn influences the grain size. Investigations of the degradation rate and the corrosion potential show that the addition of manganese improves the properties and achieves optimum values at a manganese content of $0.5 \mathrm{wt} \%$.

The property profile of extruded flat products is investigated in an original research article, and it is shown that microstructure, texture and mechanical behaviour differ significantly from rolled semi-finished products (Nienaber et al.). Three $\mathrm{Zn}$-containing magnesium alloys were extruded with different process parameters, namely ZN10, ZX10 and AZ31, and the texture, microstructure and mechanical properties were studied on the strips formed. As expected, an increase in extrusion speed results in a higher deformation temperature, which leads to an increase in dynamic recrystallization. Differences in texture were also found in the three alloys. The AZ31 showed a strong basal texture, while the ZN10 and ZX10 showed more of a tilting of the basal plane towards the extrusion direction. This is similar in round extruded profiles. Soft textures allow better formability of the flat products. It was further found that retarding the dynamic recrystallization by choosing suitable alloying elements or by varying the process parameters leads to flat products with excellent forming properties, which could also be confirmed in Erichsen tests.

A research subject that has been a focus of interest for some years now is the long-period-stacking-ordered phases (LPSO) in magnesium alloys, which are held responsible for exceptional properties of some alloys. An original research article therefore deals with short range order (SRO) clusters in a Mg97Zn1Gd2 alloy (at\%) that forms LPSO phases (Egusa et al.). GadoliniumSRO clusters were identified with high-angle annular dark-field scanning transmission electron microscopy (HAADF-STEM) and zinc atoms were detected that are constituents of these clusters. First Principle Calculations supported the identification of the atomic distribution of $\mathrm{Gd}$ and $\mathrm{Zn}$ in STEM. These investigations on atomic scales show the possibilities of electron microscopy in correlating microstructure and properties.

Another original research article dealing with LPSO phases uses a combination of in-situ neutron diffraction and acoustic emission to investigate the deformation behaviour of two $\mathrm{Mg}-\mathrm{Zn}$ Y alloys (Máthis et al.). The alloys Mg-3Y-1.5Zn and Mg-7Y-6Zn (at\%) show 32 and $85 \%$ LPSO phases in their microstructure, respectively. The investigations reveal that in both alloys the decrease in mechanical properties with an increase in temperature is only slight and that the load transfer from the magnesium matrix to the LPSO phases is the determining factor for these properties. This is particularly evident in the higher yield strength of the alloy with the higher content of LPSO phases. It turns out that twinning is the determining deformation mechanism in the alloy with low content of LPSO phases, while kinking is the determining mechanism in the other alloy. In this article, a contribution has been made to the understanding of the deformation mechanisms in LPSO alloys.

In summary, it can be stated that magnesium and its alloys are of great importance in all its variations, whether as a functionalised application (biodegradable implant material, hydrogen storage, post-Li battery, etc.), as a cast or wrought lightweight material or as a metallic matrix for hybrid materials, such as nanocomposites, laminates, etc. The wide range of papers submitted is proof of this trend, which will continue to make magnesium interesting for many applications in the future.

\section{AUTHOR CONTRIBUTIONS}

The Editorial was jointly written by the co-authors HD, DS, MP, and KK who also served as Guest Editors for the Research Topic. All authors contributed to the article and approved the submitted version.

Conflict of Interest: The authors declare that the research was conducted in the absence of any commercial or financial relationships that could be construed as a potential conflict of interest.

Copyright (c) 2021 Dieringa, StJohn, Pérez Prado and Kainer. This is an open-access article distributed under the terms of the Creative Commons Attribution License (CC $B Y)$. The use, distribution or reproduction in other forums is permitted, provided the original author(s) and the copyright owner(s) are credited and that the original publication in this journal is cited, in accordance with accepted academic practice. No use, distribution or reproduction is permitted which does not comply with these terms. 